\title{
A Survey of the Transmission-Power-Control Schemes in Wireless Body-Sensor Networks
}

\author{
Woosik Lee ${ }^{1}$, Heeyoul Kim ${ }^{2}$, Min Hong ${ }^{3}$, Min-Goo Kang ${ }^{4}$, Seung Ryul Jeong ${ }^{5}$, and Namgi Kim ${ }^{2}$ * \\ ${ }^{1}$ Research Center, Social Security Information Service, Seoul, South Korea \\ ${ }^{2}$ Department of Computer Sciences, Kyonggi University, Suwon, South Korea \\ ${ }^{3}$ Department of Computer Software Engineering, Soonchunhyang University, Asan, South Korea \\ ${ }^{4}$ Department of IT Contents, Hanshin University, Osan, South Korea \\ ${ }^{5}$ Business IT Graduate School, Kookmin University, Seoul, South Korea \\ [e-mail: ngkim@kgu.ac.kr] \\ *Corresponding author: Namgi Kim
}

Received November 23, 2017; revised February 28, 2017; accepted March 26, 2017; published April 30, 2018

\begin{abstract}
A wireless body-sensor network (WBSN) refers to a network-configured environment in which sensors are placed on both the inside and outside of the human body. The sensors are much smaller and the energy is more constrained when compared to traditional wireless sensor network (WSN) environments. The critical nature of the energy-constraint issue in WBSN environments has led to numerous studies on the reduction of energy consumption of WBSN sensors. The transmission-power-control (TPC) technique adjusts the transmission-power level (TPL) of sensors in the WBSN and reduces the energy consumption that occurs during communications. To elaborate, when transmission sensors and reception sensors are placed in various parts of the human body, the transmission sensors regularly send sensor data to the reception sensors. As the reception sensors receive data from the transmission sensors, real-time measurements of the received signal-strength indication (RSSI), which is the value that indicates the channel status, are taken to determine the TPL that suits the current-channel status. This TPL information is then sent back to the transmission sensors. The transmission sensors adjust their current TPL based on the TPL that they receive from the reception sensors. The initial TPC algorithm made linear or binary adjustments using only the information of the current-channel status. However, because various data in the WBSN environment can be utilized to create a more efficient TPC algorithm, many different types of TPC algorithms that combine human movements or fuse TPC with other algorithms have emerged. This paper defines and discusses the design and development process of an efficient TPC algorithm for WBSNs. We will describe the WBSN characteristics, model, and closed-loop mechanism, followed by an examination of recent TPC studies.
\end{abstract}

Keywords: Sensor network, wireless body sensor network, transmission power control

This work was partly supported by Institute for Information \& communications Technology Promotion(IITP) grant funded by the Korea government(MSIT) (No.2017-0-00794, Wireless Module Developments for wireless transmission of Virtual Reality contents). This work was supported by the GRRC program of Gyeonggi province. This work was supported by the Soonchunghyang University Research Fund. 


\section{Introduction}

Our society is quickly entering a super-aging era, leading to an increased interest in human health, as well as an increased demand for systems that can monitor a person's health status in real time in Internet of Things (IoT) [1]. The wireless body-sensor network (WBSN) is a human-body network that monitors health status in real time. Sensors are placed on both the inside and outside of the body and various bodily information is transmitted and received. Signals can even be sent to the nearest hospital in emergency situations. However, because WBSNs must operate in and on the human body, unlike traditional wireless sensor networks (WSNs), sensor size in WBSNs is extremely small and energy resources are limited. Therefore, it is crucial to reduce the energy consumption of sensors in WBSN environments as Fig. 1.

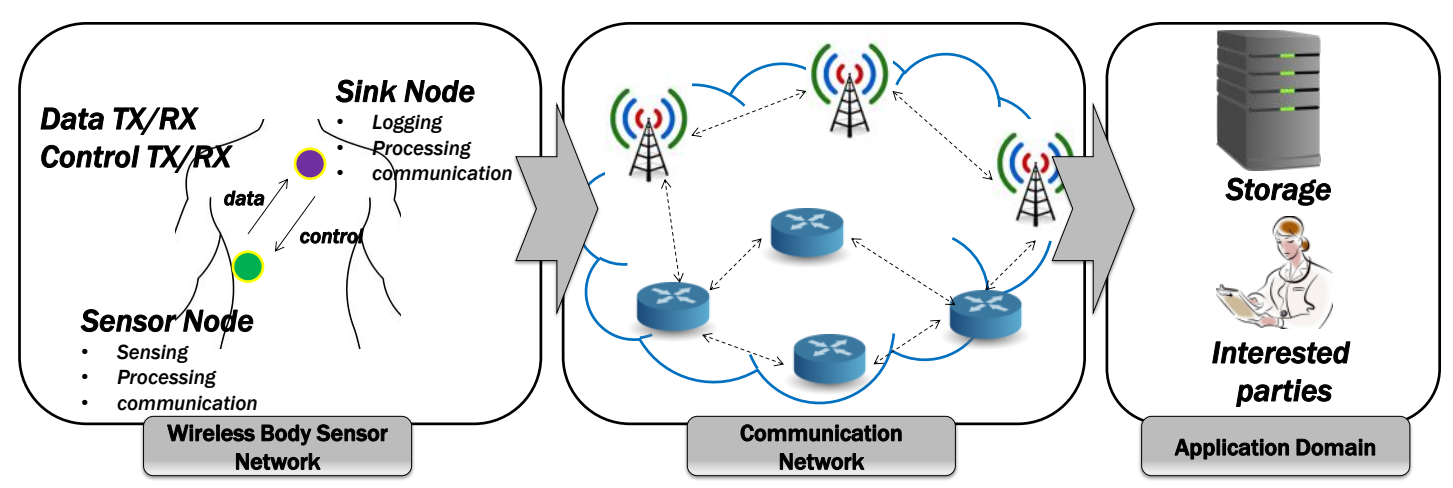

Fig. 1. Wireless Body Sensor Network Architecture

The transmission-power-control (TPC) algorithm is one of the techniques used for the reduction of sensor energy consumption in WBSN environments. The TPC algorithm reduces the energy consumption that occurs during sensor communication by adjusting the transmission-power level (TPL) of the radio modules in the sensors. To elaborate, WBSN sensors use $\mathrm{CC} 1000$ or CC2420 radio modules for communication. The TPLs of the CC1000 and $\mathrm{CC} 2420$ are 21 and 31, respectively. When a high-TPL communication occurs, the communication range and data-success rate are increased, but there is a corresponding disadvantage of increased energy consumption. When a low-TPL communication occurs, the communication range and data-success rate may suffer, but the sensors operate with higher energy efficiency, meaning sensor lifespan can be extended by using a TPL that consumes minimal energy. This tradeoff must be balanced so that a high data-success rate with an appropriate communication range is still guaranteed. The TPC algorithm is a technique that aids in the efficient selection of TPLs for sensor radio modules.

Unlike in traditional WSN environments, the wireless channel in WBSN environments is unstable and dynamic. This is due to the possibility of continuous communication in the absence of sudden incidents, such as changes in sensor location or diastrophism, because the communication objective is a common goal and sensors are placed at fixed locations in WSN environments. In contrast, the sensors in WBSN environments are disrupted by communication channels in real time based on parts of the human body. Furthermore, channel status becomes unstable based on a person's movements, particularly when a person makes prominent movements. There are also cases where a channel changes from an indoor 
environment to an outdoor environment because of a person's movements. Therefore, a WBSN environment requires the consideration of multiple factors, such as sensor placement and an individual's movements.

We examine channel changes in WBSN-channel environments based on radio modules, sensor locations, and a person's location, which are factors that must be considered for the development of an efficient TPC algorithm. Additionally, we introduce the model and closed-loop mechanism for the design of a TPC algorithm. Finally, a comparative analysis is performed using the most recent TPC algorithms.

Therefore, this paper's contributions are as follows. First, we show the characteristics of the wireless body sensor network, which is essential for WBSN application design. Moreover, we analyze various WBSN elements such as the sensor device specifications, human movement and sensor deployment. Second, we define a model of the transmission power control algorithm to reduce the power consumption of sensor devices. Third, we introduce the closed-loop mechanism of the transmission power control algorithm for controlling the energy level of radio chips. Lastly, we compare recent TPC algorithm researches.

This remainder of this paper is organized as follows. Section 2 examines the characteristics of WBSNs. Section 3 examines the components of a TPC algorithm model. Next, we introduce closed-loop mechanism. Section 5 compares the most recent TPC algorithms. The final section summarizes this study and introduces plans for future studies.

\section{Characteristics of Wireless Body-Sensor Networks}

This section examines the characteristics of WBSN environments. First, the sensors and radio modules that are frequently used in WBSN environments will be examined through a comparison of commercial sensor nodes. Next, the received signal-strength indicator (RSSI) and packet-delivery ratio (PDR) based on a person's movements will be examined using the CC1000 and CC2420, which are the main radio modules used in WBSN environments. Finally, an RSSI-distribution analysis will be performed based on the locations of sensors that are placed in the human body.

For WBSNs, representative commercial sensor nodes, such as BTnode [2], Tmote Sky [3], Mica2 [4], Mica [5], Mica2 Dot [6], TelosB [7], and IRIS [8], can be installed. The radio modules, frequencies, output power, and TPL of each sensor vary according to their objectives. BTnode, Mica2, Micaz, Mica2 Dot, and IRIS are used with the ATmega 128L Microcontroller [9], while Tmote Sky and TelosB are used with the MSP 430 Microcontroller [10]. The most common communication chips in the sensors are the CC1000 [11] and CC2420 [12]. BTnode, Mica2, and Mica2 Dot use the CC1000 communication chip, while Tmote Sky, Micaz, TelosB, and IRIS use the CC2420 communication chip. The frequencies of the sensors that use the CC1000 communication chip range from $433-916 \mathrm{MHz}$ and their output power ranges from-20 to $10 \mathrm{dBm}$. The frequency and output power of the sensors that use the CC2420 communication chip are $2.4 \mathrm{GHz}$ and range from -25 to $0 \mathrm{dBm}$, respectively. Table1 presents a comparison of the commercial sensor nodes that are frequently used in WBSN environments. 
Table 1. Comparison of Commercial Sensor Nodes

\begin{tabular}{|c|c|c|l|c|}
\hline Sensor Node & Radio Module & Frequency & Output Power & TPL \\
\hline \hline BTnode & Chipcon CC1000 & $433-916 \mathrm{MHz}$ & -20 to $10 \mathrm{dBm}$ & 0 to 21 \\
\hline Tmote Sky & Chipcon CC2420 & $2.4 \mathrm{GHz}$ & -25 to $0 \mathrm{dBm}$ & 0 to 31 \\
\hline Mica 2 & Chipcon CC1000 & $433-916 \mathrm{MHz}$ & -20 to $10 \mathrm{dBm}$ & 0 to 21 \\
\hline Micaz & Chipcon CC2420 & $2.4 \mathrm{GHz}$ & -25 to $0 \mathrm{dBm}$ & 0 to 31 \\
\hline Mica2 Dot & Chipcon CC1000 & $433-916 \mathrm{MHz}$ & -20 to $10 \mathrm{dBm}$ & 0 to 21 \\
\hline TelosB & Chipcon CC2420 & $2.4 \mathrm{GHz}$ & -25 to $0 \mathrm{dBm}$ & 0 to 31 \\
\hline IRIS & Chipcon CC2420 & $2.4 \mathrm{GHz}$ & -25 to $0 \mathrm{dBm}$ & 0 to 31 \\
\hline
\end{tabular}

In a WBSN, a person's movements are divided into the three main categories of standing, walking, and running. Standing is when a person is standing still without any movement, walking is when a person is moving at an average speed of approximately $5.0 \mathrm{~km} / \mathrm{h}$, and running is when a person is moving at an average speed of $15.0 \mathrm{~km} / \mathrm{h}$. In a WBSN environment, a person's movements are typically a mixture of standing, walking, and running, meaning they change dynamically. Channel status is worse when a person is walking compared to when they are standing, and is even worse when running compared to walking. This is because parts of the human body increasingly disrupt radio waves as a person's movements become more exaggerated and because more communication obstacles are typically present during movement.

Fig. 2 presents the RSSI and PDR changes in the CC1000 and CC2420 radio modules based on a person's standing, walking, and running movements. The bars in the graph represent the PDR values and the dots represent the RSSI values. When an analysis was performed from an RSSI/PDR perspective, both the RSSI and PDR values were improved when the TPL value was increased for both the $\mathrm{CC} 1000$ and $\mathrm{CC} 2420$ radio modules. This is because the transmission range was extended and the output power was increased at a higher TPL.

When the test results are analyzed from the perspective of a person's movements, the range of RSSI values is narrow when a person is in a stable standing state. However, when the person's movements became more dynamic in the walking or running state, the deviation in RSSI values increased, indicating that the WBSN channel is disturbed and becomes unstable as a person's movements become more dynamic.

When the test is analyzed from the perspective of the radio modules, the RSSI-value distribution of the $\mathrm{CC} 2420$ radio module is wider than that of the $\mathrm{CC} 1000$ radio module. This is because the CC1000 frequency range is lower than the 2.4-GHz frequency of the CC2420, which results in a higher radio-wave refractive index. The CC2420-frequency linearity is also higher. The data transmission of the higher-linearity radio waves suffers in the presence of obstacles and high levels of refraction. 

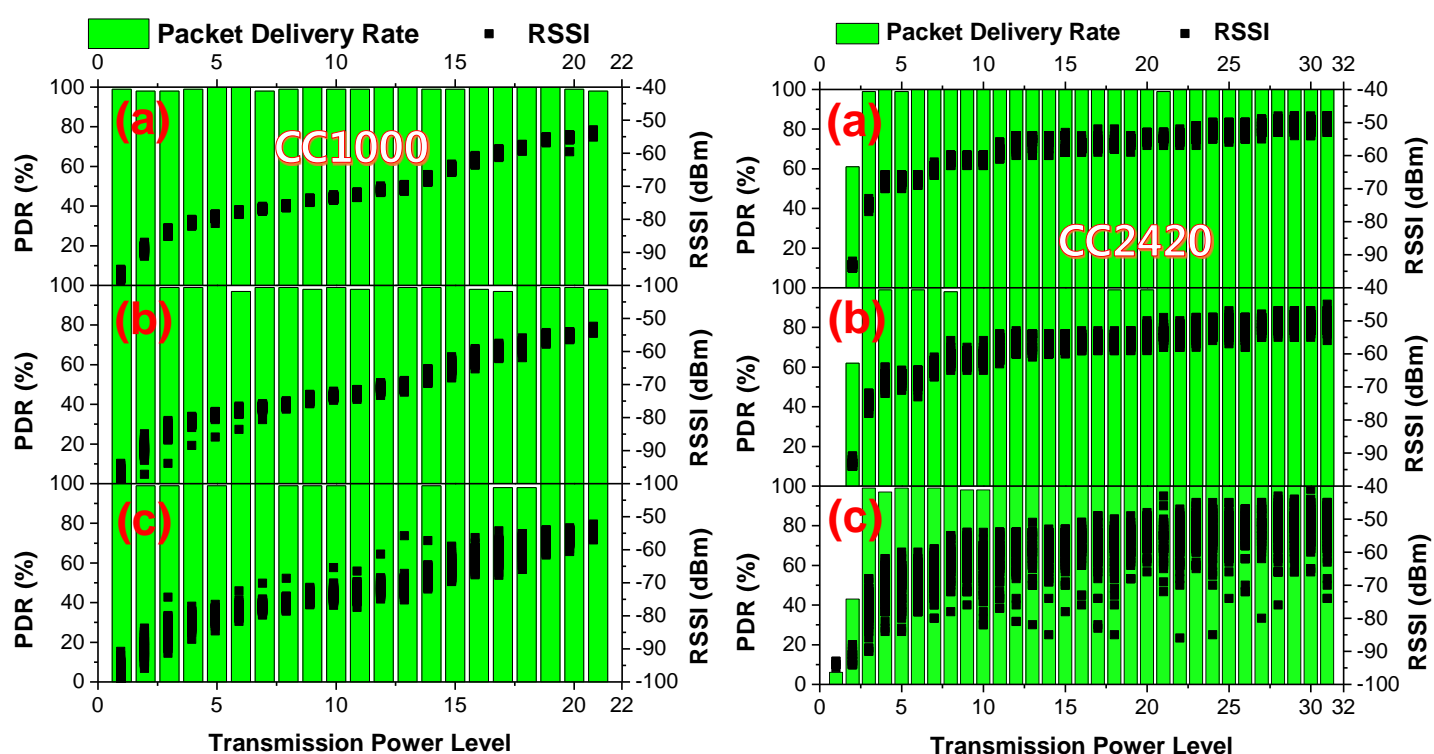

Fig. 2. Received Signal Strength Indicator (RSSI) and Packet Delivery Ratio (PDR) Values for the CC1000 (left) and CC2420 (right) for the Standing, Walking, and Running Categories: (a) Standing, (b) Walking, and (c) Running [13]

In a WBSN, small sensors, such as those for electrocardiograms (ECG), electroencephalograms (EEG), blood-oxygen-saturation (SpO2), and blood-pressure measurements, are placed in various parts of the human body to detect body-status changes in real time. The detected values are then delivered to the sensors that serve as central gateways. The majority of the sensors that serve as gateways are located in a favorable positions for the reception of data from all of sensors and the collection of data from bodily signal sensors. However, if a body part disrupts data transmission and reception, the data-reception rate will suffer.

Fig. 3 presents the RSSI distributions that occurred during the placement of the gateway sensor nodes in the chest for sensors in the following ten body parts: left ankle, right ankle, left thigh, right thigh, stomach, back, left wrist, right wrist, left forearm, and right forearm. The graph on the left in Fig. 3 shows how the RSSI distribution changes with time and the graph on the right shows the RSSI distribution based on the body-placement locations.

The RSSI-distribution graph based on time shows that the value of the received-power strength for the chest-stomach sensor is higher than that of the sensors that were placed in other parts of the body. This is because this sensor is placed in the location that is nearest to the chest-sensor node, which is where the gateway sensor is, and because a line-of-sight that is not interrupted by any obstacles is maintained between the transmitter and the receiver. However, the chest-ankle sensor and the chest-forearm sensor both display lower received-power-strength results. The low received-power strength of the chest-ankle sensor is a result of signal-attenuation that is attributable to the long communication distance to the transmitter. Although it seems likely that signal-attenuation would not occur in the relatively short distance to the chest-forearm sensor, it does occur because a person's arms may move even when they are standing in a fixed location, meaning the orientation of the body sensor may differ from that of the transmitter sensor. 
In the RSSI graph based on sensor placement in Fig. 3, the RSSI deviation of the chest-stomach sensor is less than that of the wrist, thigh, back, ankle, and forearm sensors. The lowest RSSI value and highest deviation occur for the chest-forearm sensor. The RSSI deviation of the chest-back sensor is extremely small and its RSSI value is favorable when compared to the chest-forearm and chest-ankle sensors. This is because the sensor device in the back is always in a fixed location, similar to the sensor in the stomach, unlike the forearms or ankles, where movement is always present, even in a static environment. However, the received-power strength is lower than that of the stomach sensor because the communication distance from the transmitter is longer and the radio waves are disrupted by the human body.
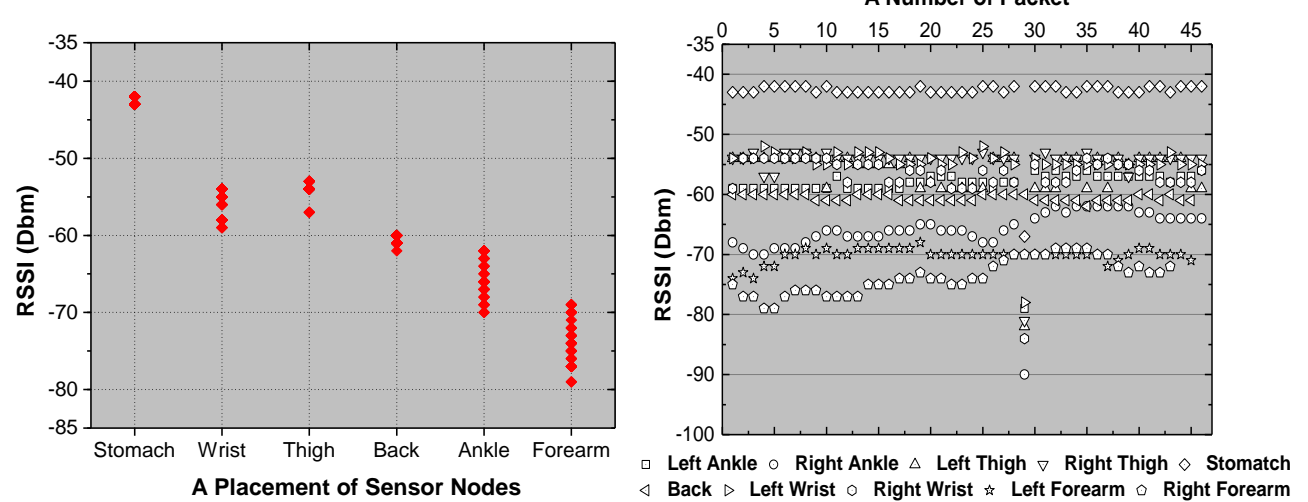

Fig. 3. Received Signal Strength Indicator (RSSI) based on Sensor Placements in the Ankle, Thigh, Stomach, Back, Wrist, and Forearm [14]

\section{Model for Transmission-Power Control}

This section introduces a model for the use of a TPC algorithm to determine the optimal transmission power for sensors in the WBSN. The Model for Transmission-Power Control involves the following components:

- Target RSSI Margin: RSSI range of the appropriate transmission power

- Target RSSI Point: RSSI point of the most appropriate transmission power

- Target RSSI MAX: maximum RSSI point of the appropriate transmission power

- Target RSSI MIN: minimum RSSI point of the appropriate transmission power

- Standing Value Range: the distribution range of average RSSI values while a person is standing in a WBSN environment.

- Walking Value Range: the distribution range of average RSSI values while a person is walking in a WBSN environment.

- Running Value Range: the distribution range of average RSSI values while a person is running in a WBSN environment.

Fig. 4 presents the TPC model based on the Target RSSI Margin, Target RSSI Point, Target RSSI MAX, and Target RSSI Min components. The $x$-axis in the model is the TPL and the $y$-axis is the RSSI value.

The movement principle of the proposed TPC model is that the sensor TPL does not need to be controlled if the current RSSI value is within the Target RSSI Margin. However, if the 
current RSSI value is higher than the Target RSSI MAX value, the TPL must be reduced so that the RSSI value falls within the Target RSSI Margin. Similarly, if the current RSSI value is lower than the Target RSSI MIN value, the TPL must be increased so that the RSSI value falls within the Target RSSI Margin.

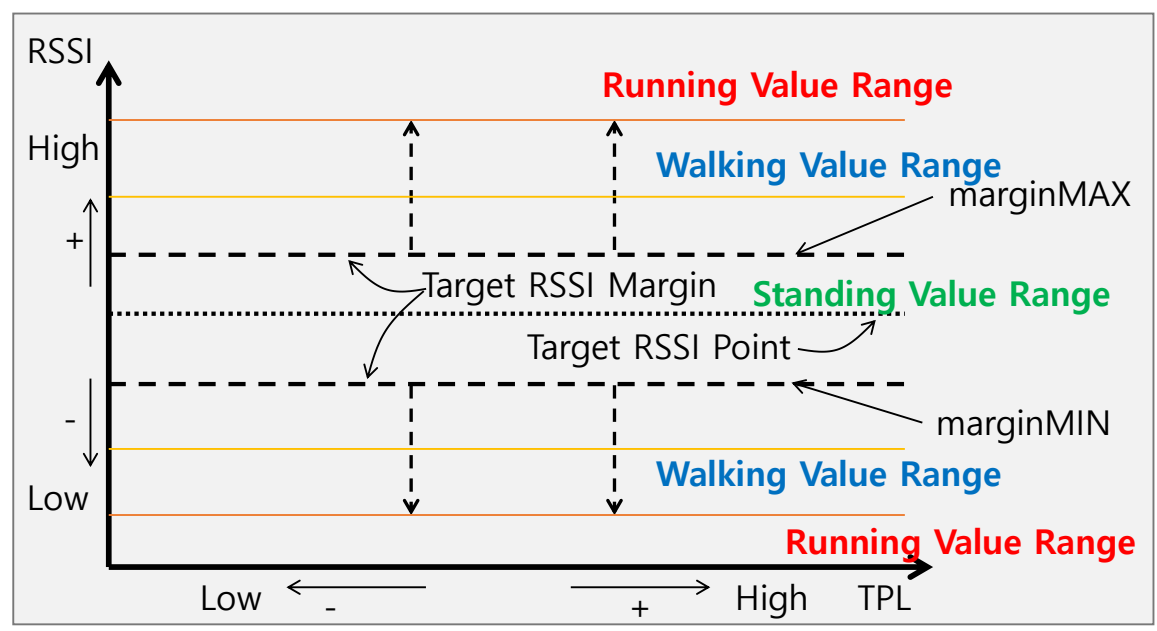

Fig. 4. Model of Transmission-Power Control (TPC) in a Wireless Body-Sensor Network (WBSN)

\section{Closed-Loop Mechanism for Transmission-Power Control}

This section introduces the closed-loop mechanism that is designed so that sensors perform transmission and reception based on the TPC model and renew their current-channel status, then send and receive feedback messages based on the channel status. The transmitter of the closed-loop mechanism is the sensor that collects body data and the receiver is the sensor that serves as a gateway.

The transmitter collects body data in real time and transmits the data from the current TPL to the gateway sensor. The gateway sensor then measures the RSSI value, which is the transmission-power strength that is measured when the data are received. The current RSSI value is assessed to determine if it is within the Target RSSI Margin based on the TPC model that was described in Section 2. If the current RSSI value is within the Target RSSI Margin, the gateway node will not send a control message to the transmitter, because it does not need to update the TPL.

However, if the current RSSI value is outside the Target RSSI Margin, the gateway node will find a new appropriate TPL value using the TPC algorithm. The transmitter will then be sent a control message that contains the new TPL value. The transmitter receives the control message and updates the TPL if the TPL value in the received control message is different from the current TPL value. Messages are then transmitted using the updated TPL. Fig. 5 presents the overall structure of the closed-loop mechanism. 


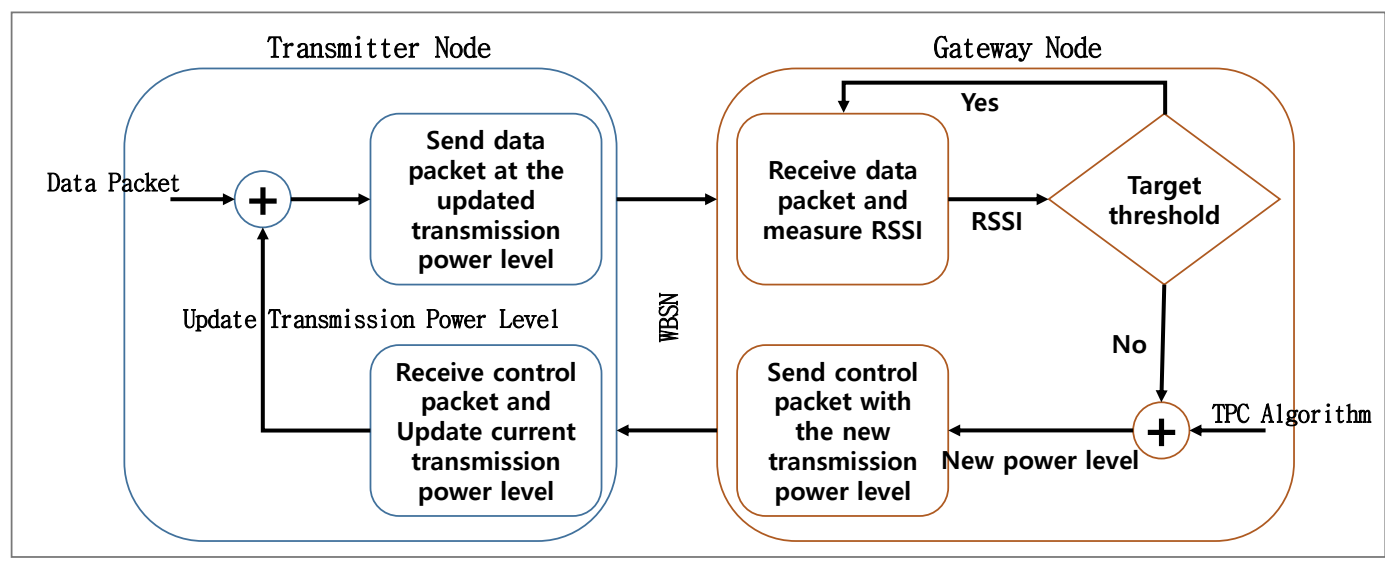

Fig. 5. Closed-loop Mechanism of a Wireless Body-Sensor Network (WBSN)

\section{Transmission-Power Control Scheme}

This section examines the most recent algorithms used for TPC in WBSN environments. First, the following TPC algorithms will be examined: Linear [15], Binary [16], and Dynamic [17]. Linear TPC is an algorithm that controls transmission power by using four fundamental arithmetic operations. The Linear TPC algorithm adjusts the TPL by one level at a time based on the current-channel status. For example, if the RSSI value, which is the received power-strength value that represents the current channel, is higher than the Target RSSI Margin MAX, the TPL will be lowered by one level. If the current TPL is 22, it will then be changed to 21. Alternatively, if the current-channel RSSI value is lower than the Target RSSI Margin MIN, the TPL will be increased by one level. For example, if the current TPL level is five, the data will be transmitted using a TPL of the next level, which is level six. This process is repeated until the optimal TPL is found. Therefore, a large amount of time is required to find the optimal transmission power compared to the other algorithms when the channel is stable. However, the Linear TPC algorithm is more efficient than the other algorithms in a continuously changing environment, even for TPLs with consistent RSSI values, where the channel is unstable. This is because the received-power strength can be continuously placed within the Target RSSI Margin by keeping the power near an efficient TPL. This is easily accomplished because the Linear TPC algorithm, which finds the TPL before the channel becomes unstable, adjusts the TPL one level at a time. Furthermore, the Linear TPC algorithm, which keeps the TPL near the Target RSSI Margin, displays a high data-success rate and a low data-loss rate.

Unlike the Linear TPC algorithm, the Binary TPC algorithm adjusts the TPL exponentially. It is extremely efficient because it can quickly find the optimal TPL when the channel is stable. However, when the channel is unstable, an oscillation effect may occur, making it impossible to find an appropriate TPL because the initial TPL that was predicted continues to miss and the new TPL changes exponentially. Therefore, the Binary TPC algorithm is extremely inefficient when the channel is unstable.

The Dynamic TPC algorithm finds the optimal TPL based on a primary equation. Here, the TPC algorithm is based on an equation for the attainment of a formula for the Dynamic TPC algorithm, which requires two separate data collections. The equation's slope and constant are 
obtained based on two collected RSSI values. After a $Y=\mathrm{a} X+\mathrm{c}$ equation is obtained using the slope and constant, the TPL can be found instantly using the equation. Because the Dynamic TPC algorithm is accurate in an environment where the channel status is stable, the optimal TPL can be found quickly. However, when the channel status is unstable, a precise TPL cannot be found due to an inaccurate equation and large amounts of energy are wasted.

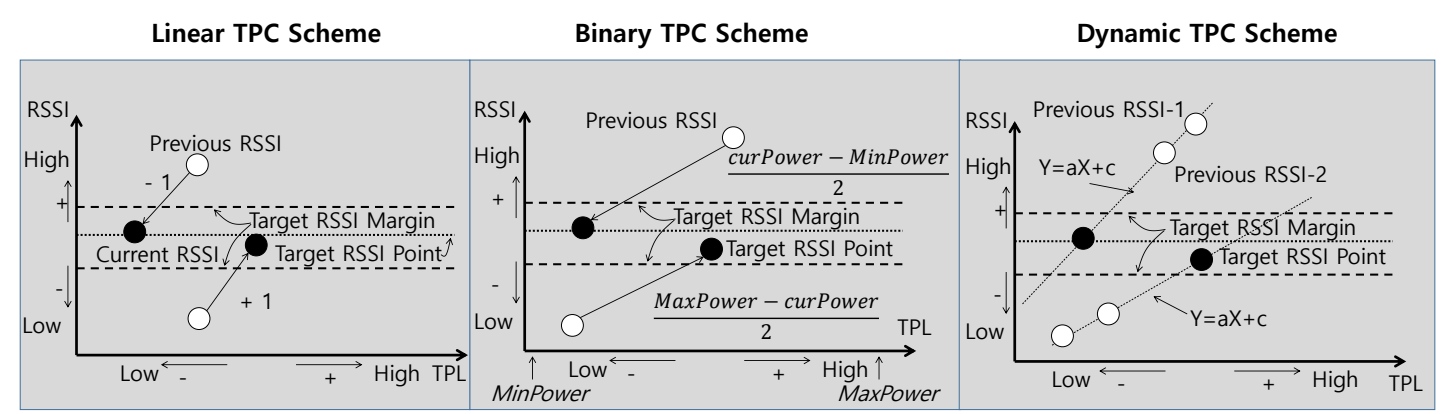

Fig. 6. Linear, Binary, and Dynamic Transmission-Power Control (TPC) Schemes

RSSI/link-quality-indicator (LQI)-based TPC [18] is a technique that controls sensor transmission power by considering link-status information (the aforementioned LQI) instead of only considering channel status by using the RSSI value. To this end, the author of [18] attached sensors to a person's wrists and back to measure RSSI values. Additionally, the data-loss rate was derived from the channel status based on the LQI value that represents link quality. Tests that controlled TPL by considering both the RSSI and LQI values demonstrated that this technique is superior to using RSSI alone.

The Hybrid TPC algorithm [19] is a TPC algorithm in which both stable and dynamic environments are considered. It uses the Binary TPC algorithm in stable environments and the Linear TPC algorithm in dynamic environments. The decision of which algorithm to use is made in real time and the Hybrid TPC algorithm evaluates the current-channel environment based on changing RSSI values.

In [20], an innovative control-packet adjustment method that can reduce control-packet waste was proposed in order to reduce the amount of energy that is wasted in a WBSN where the channel environment changes dynamically. The proposed control-packet adjustment method continuously calculates average RSSI values in real time. A new TPL value is found when the difference between the average RSSI value and the current RSSI value exceeds a certain threshold, thereby preventing unnecessary control-packet waste and increasing sensor lifespan.

The authors of [21] proposed an adaptive channel-gain predictor that can find the optimal margin of transmission power model by using an adaptive fade-margin estimator that can measure a channel's radio-wave characteristics in real time. It can also predict radio-wave conditions that change based on a person's movements. The channel status was predicted using the system proposed in [21]. The authors measured the energy by using a sensor that utilizes the CC2420-transmission chip. They measured the energy-consumption values from both the transmission messages and control messages. Upon a comparison of the results with those of the existing adaptive-TPC(ATPC) algorithm, for which a real-world experiment was conducted, the energy consumption was reduced by an average of $16 \%$.

The ACC-based TPC algorithm [22] utilizes a modified version of the basic closed-loop mechanism. Because the existing mechanism operates by measuring the current-channel 
condition based on RSSI values, it only considers systemic actions that respond instantly to channel changes, without the use of control-message delay or other such actions. However, because the ACC-based TPC algorithm must consider the acceleration value in addition to the RSSI, the acceleration value is measured at the same point that the RSSI is measured.

In [23], an algorithm that controls the TPL by creating an equation with the channel-analysis value of a dynamic WBSN environment was proposed. The proposed algorithm showed that the received-power strength in the standing and running states differed based on the linear-equation type when the sensor was placed on the chest, stomach, or arm in an indoor or an outdoor environment. These findings were used to propose an equation-based TPC algorithm. The proposed TPC algorithm finds an efficient TPL by obtaining an average RSSI value based on the slope of linear equation, dividing the difference between the average and current RSSI values by the slope in real time, and then adding the current power value to obtain a new TPL.

The motion-aware-TPC (M-TPC) algorithm [24] was proposed as a TPC algorithm that is based on a person's movements in the WBSN environment. First, the author investigated the ways in which channels change based on a person's movements in the WBSN environment in order to determine the correlation between channel changes and a person's movements. The author revealed a strong correlation between channel-path loss and bodily movements. A human-motion recognition algorithm that was configured previously was used to determine if a person was walking or running. The TPC algorithm was then applied. RSSI and TPL values were measured in real time and a new TPL was obtained by using the M-TPC algorithm. The new value was then shared with nearby sensors to facilitate communications that used the minimum adequate transmission power.

In [25], the author proposed a TPC algorithm that controls the TPL based on a person's movements in a WBSN environment. They conducted an experiment by placing sensors that transmit data periodically on a wall and putting the receivers on a person's wrists. The proposed method collected data from the sensors in real time to find the location of the current cycle. If the cycle value was greater than an "a" value that was set in advance, the algorithm calculated the average cycle and a prediction value for the person's movement. The optimal TPL value was identified based on the value that was measured subsequently. The new TPL value was then transmitted and received by the sensors.

The author of [26] proposed a feedback-based TPC algorithm for efficient control of the TPL in a WBSN. The proposed algorithm updated the RSSI value in real time by using the difference between the current channel's RSSI value and the average RSSI value, based on two parameters, $a$ and $b$. After deriving the minimum power through a new formula that calculates the TPL, the current power was updated. The author of [27] proposed a system that could not be resolved using existing TPC algorithms in a dynamic WBSN environment. The author analyzed an acceleration value in real time and extracted its features. They then determined if the status was moving or static based on the features. If the status was moving, then the acceleration-based TPC algorithm was used. Otherwise, the RSSI/LQI-based TPC algorithm was used. Upon the discovery of a strong correlation between the acceleration value and the RSSI value through various tests, the author controlled the current TPL based on the number of values that exceeded the acceleration threshold.

The author of [28] proposed a new method in an effort to resolve the unstable channel status that occurs when the distance between the sensors in the WBSN is greater than 140. The proposed algorithm involved the placement of a relay node between the sensors if the distance between them became too large. This node played a mediating role and TPL could be 
controlled accordingly. The channel status is unfavorable when a receiver, relay base, and transmitter are present, thereby preventing direct communication between the transmitter and receiver, meaning the relay base was used to transmit the status instead. To facilitate this mechanism, the author proposed an algorithm that connects the transmitter and receiver directly when the channel status is favorable. Otherwise, communication was performed using a relay base in one of the following three modes: transmitter-relay base, relay base-receiver, and transmitter-receiver.

In [29], Gaussian-process regression (GPR) was performed on data collected by the sensors. The proposed TPC algorithm controls the transmitter's TPL based on the current level. In the proposed algorithm, the transmitter first sends the data to the receiver at maximum power. The receiver then measures the received RSSI value. If the value exceeds a certain threshold, the receiver sends the received-power strength back to the transmitter. The transmitter uses the data from the receiver as training data, and then predicts the transmission power of the next transmission.

The MAPM (Movement-based Adaptive Prediction Mechanism) [30] predicts a person's movements and controls sensor TPL based on the predictions. In the MAPM, instead of sending feedback from the receiver to control the transmission power, the receiver uses the GPR to assess pattern changes based on a person's movements in order to prevent transmission-power-based energy waste. To this end, the author proposed a prediction algorithm using covariance, mean, and likelihood functions to express transmission-power prediction through a formula. The proposed algorithm was tested using an Android phone and it displayed outstanding performance.

Table 2 presents the most recent TPC algorithms that were categorized and analyzed based on the radio-chip used, movement, placement, and evaluation parameters. The majority of transmission-power studies in body-sensor-network environments use the CC1000 or CC2420 radio modules, and most are conducted based on a dynamic-channel status, such as walking or running, rather than a stable-channel status, such as standing. Most studies conducted tests with consideration for either a line-of-sight state, where obstacles are not present between the transmitter and receiver sensors, or a non-line-of-sight state, where obstacles are present. Finally, the majority of the studies considered packet-transmission success rate and energy consumption as evaluation factors.

Table 2. Comparison of Recent Transmission-Power Control (TPC) Schemes

\begin{tabular}{|c|c|c|c|c|c|c|}
\hline Ref. & Year & Algorithm & Radio Chip & Movements & Placement & Evaluation \\
\hline \hline$[17]$ & 2010 & Dynamic TPC & CC1000 & $\begin{array}{c}\text { Sitting, } \\
\text { Walking, } \\
\text { Standing, }\end{array}$ & $\begin{array}{c}\text { Stomach, } \\
\text { Forearm, } \\
\text { Wrist }\end{array}$ & $\begin{array}{c}\text { PDR, } \\
\text { Energy }\end{array}$ \\
\hline$[18]$ & 2013 & RSSI/LQI-based TPC & CC2420 & $\begin{array}{c}\text { Slow Walk, } \\
\text { Middle Walk, } \\
\text { Fast Walk }\end{array}$ & $\begin{array}{c}\text { Wrist, } \\
\text { Waist }\end{array}$ & PDR, TPL \\
\hline$[19]$ & 2014 & Hybrid TPC & CC1000 & $\begin{array}{c}\text { Standing, } \\
\text { Walking, } \\
\text { Running }\end{array}$ & $\begin{array}{c}\text { Chest, } \\
\text { Stomach, } \\
\text { Back }\end{array}$ & PDR, \\
Energy
\end{tabular}




\begin{tabular}{|c|c|c|c|c|c|c|}
\hline [21] & 2014 & On-Off Body TPC & CC2420 & $\begin{array}{c}\text { Standing, } \\
\text { Sitting, } \\
\text { Walking, } \\
\text { Running } \\
\end{array}$ & $\begin{array}{c}\text { Arm, Wrist, } \\
\text { Waist, } \\
\text { Ankle, Back, } \\
\text { Head } \\
\end{array}$ & Energy \\
\hline [22] & 2015 & ACC-based TPC & CC1000 & $\begin{array}{l}\text { Standing, } \\
\text { Walking, } \\
\text { Running }\end{array}$ & $\begin{array}{c}\text { Chest, } \\
\text { Stomach, } \\
\text { Back, Arm }\end{array}$ & $\begin{array}{l}\text { PDR, } \\
\text { Energy }\end{array}$ \\
\hline [23] & 2015 & Equation-based TPC & CC1000 & $\begin{array}{l}\text { Standing, } \\
\text { Running }\end{array}$ & $\begin{array}{l}\text { Stomach, } \\
\text { Back, Arm }\end{array}$ & Energy \\
\hline [24] & 2015 & Motion-aware TPC & CC2530 & $\begin{array}{l}\text { Walking, } \\
\text { Running }\end{array}$ & Wrist, Waist & PDR, TPL \\
\hline [25] & 2016 & $\begin{array}{c}\text { Human-motion-based } \\
\text { TPC }\end{array}$ & CC2420 & Sitting & $\begin{array}{l}\text { Wrist, } \\
\text { On the Wall }\end{array}$ & $\begin{array}{l}\text { PDR, } \\
\text { Energy }\end{array}$ \\
\hline [26] & 2016 & $\begin{array}{c}\text { Energy-efficient-based } \\
\text { TPC }\end{array}$ & CC2420 & Walking & $\begin{array}{l}\text { Wrist, Hip, } \\
\text { Chest }\end{array}$ & $\begin{array}{l}\text { PDR, } \\
\text { Energy }\end{array}$ \\
\hline [27] & 2016 & ACC-assisted TPC & $\mathrm{CC} 2420$ & $\begin{array}{l}\text { Sitting, } \\
\text { Walking }\end{array}$ & Wrist, Waist & $\begin{array}{l}\text { PDR, } \\
\text { Energy }\end{array}$ \\
\hline [28] & 2017 & Relay-aided TPC & CC2420 & Standing & $\begin{array}{l}\text { Chest, } \\
\text { Stomach, } \\
\text { Ankle }\end{array}$ & $\begin{array}{l}\text { PDR, } \\
\text { Energy }\end{array}$ \\
\hline [29] & 2017 & Gait-cycle-driven TPC & CC2420 & $\begin{array}{l}\text { Standing, } \\
\text { Fast Walk, } \\
\text { Normal Walk }\end{array}$ & $\begin{array}{c}\text { Ankle, } \\
\text { Pants pocket }\end{array}$ & $\begin{array}{l}\text { PDR, } \\
\text { Energy }\end{array}$ \\
\hline [30] & 2017 & MAPM TPC & Smartphone & $\begin{array}{l}\text { Standing, } \\
\text { Fast Walk, } \\
\text { Normal Walk }\end{array}$ & $\begin{array}{l}\text { Wrist, } \\
\text { Waist }\end{array}$ & TPL \\
\hline
\end{tabular}

\section{Conclusion}

The sensors in WBSN environments are smaller than those in traditional sensor networks, which results in a greater number of constraints in terms of resources and energy. This paper analyzed the characteristics of WBSN environments from various perspectives, including sensor devices, a person's movements, and sensor placement. Additionally, this paper introduced TPC algorithms that are widely used for the reduction of sensor energy consumption, as well as the closed-loop mechanism that enables the exchange of transmission-power information between the transmitter and receiver sensors through a feedback method.

The main types of TPC algorithms that were discussed are the linear, binary, and dynamic types. This paper introduced the basic foundational TPC algorithms, as well as various recent TPC algorithms that were derived from the basic algorithms, such as the Hybrid, ACC-based, Human-motion, and Relay-aided algorithms.

Through the analysis of various recent TPC algorithms, this paper gives the insight that the paradigm of TPC algorithms in the near future will be changed from feedback mechanism to self-control mechanism based on big-data and artificial intelligence. Moreover, the general 
analysis of WBSN environments in this paper will become a significant reference for the formulation of algorithms that extend the lifespan of sensors in the future.

In the future, our research team will conduct a study on the linkage of various objects, body-sensor networks, and the expanding platforms of the fourth industrial revolution.

\section{References}

[1] J.S. Song, A.Kunz, M. Schmidt, and P. Szczytowski, "Connecting and Managing M2M Devices in the Future Internet," Mobile Networks and Applications, pp. 4-17, Feb. 2014.

Article (CrossRef Link)

[2] BTnode, http://www.btnode.ethz.ch/

[3] Tmote Sky, http://wirelesssensornetworks.weebly.com/1/post/2013/08/tmote-sky.html

[4] Mica2, http://www.snm.ethz.ch/Projects/Mica2

[5] Micaz, http://www.memsic.com

[6] Mica2Dot, http://www.willow.co.uk/html/mpr5x0-_mica2dot_series.php

[7] TelosB, http://www.willow.co.uk/html/telosb_mote_platform.php

[8] IRIS, http://www.xbow.com/Products/productdetails.aspx?sid=264

[9] Tmega 128, Available at http://www.microchip.com/wwwproducts/en/ATmega128

[10] MSP 430, http://www.ti.com/microcontrollers/msp430-ultra-low-power-mcus/overview.html

[11] CC1000, http://www.ti.com/lit/ds/symlink/cc1000.pdf

[12] CC2420, https://www.ti.com/lit/ds/symlink/cc2420.pdf

[13] Woosik Lee, Min Choi, and Namgi Kim, "Different Characteristics of Radio Modules in Wireless Body Sensor Network Systems," Lecture Notes in Computer Science, pp. 308-314, Sep. 2012. Article (CrossRef Link)

[14] Woosik Lee, Min Choi, and Namgi Kim, "Experimental Link Channel Characteristics in Wireless Body Sensor Systems," IEEE ICOIN, pp. 374-378, Feb. 2012. Article (CrossRef Link)

[15] D. Knuth, "The Art of Computer Programming: Volume 3: Sorting and Searching (2nd Edition)," Addison-Wesley Professional, 1997. Article (CrossRef Link)

[16] T. H. Cormen, C.E. Leiserson, and R. L. Rivest, "Introduction to Algorithms," MIT Press and McGraw-Hill $1^{\text {st }}$ edition, 1990.

[17] M. Quwaider, J. Rao, and S. Biswas, "Body-Posture-Based Dynamic Link Power Control in Wearable Sensor Networks," IEEE Communications Magazine, pp. 134-142, July 2010. Article (CrossRef Link)

[18] S. Kim, S. Kim, and D.-S. Eom, "RSSI/LQI-based Transmission Power Control for Body Area Networks in Healthcare Environment," IEEE Journal Biomedical Health Information, pp. 561-571, May. 2013. Article (CrossRef Link)

[19] W.S. Lee, B.D. Lee, and N. Kim, "Hybrid Transmission Power Control for Wireless Body Sensor Systems," International Journal of Distributed Sensor Networks, pp. 1-9, Oct. 2014. Article (CrossRef Link)

[20] Gangman Yi, Daeun Yu, and Namgi Kim, "Adjusting Control Packet Transmission Intervals in Low Power Sensor Systems," International Journal of Distributed Sensor Network, pp. 1-8, Aug. 2014. Article (CrossRef Link)

[21] F. Di Franco, Y. Ge, and I. Tinnirello, "On-body and Off-body Transmit Power Control in IEEE 802.15.6 Scheduled Access Networks, ” in Proc. IEEE PIMRC, pp. 1254-1258, Sept. 2014. Article (CrossRef Link)

[22] Woosik Lee, Byoung-Dai Lee, and Namgi Kim, "An Accelerometer-Assisted Power Management for Wearable Sensor Systems," KSII Transactions on internet and information systems, pp. 318-330, Jan. 2015. Article (CrossRef Link)

[23] Namgi Kim, Jin-a Hong, and Byoung-Dai Lee, "Equation-Based Transmission Power Control for Wearable Sensor Systems," IEICE Trans, fundamentals, pp. 1558-1561, July 2015. Article (CrossRef Link) 
[24] W. Zang and Y. Li, "Motion Aware Transmission Power Control Scheme in Wireless Body Area Network, " in Proc. IEEE Workshop SIPS, pp. 1-5, Oct. 2015. Article (CrossRef Link)

[25] G. Newell and G. Vejarano, "Human-motion based Transmission Power Control in Wireless Body Area Networks," IEEE WF-IoT, pp. 277-282, Dec. 2016. Article (CrossRef Link)

[26] A.H. Sodhro, Y. Li, and M.A. Shah, "Energy-efficient Adaptive Transmission Power Control for Wireless Body Area Networks," IET Communications, pp. 81-90, Feb. 2016. Article (CrossRef Link)

[27] W. Zang, S. Zhang, and Y. Li, "An Accelerometer-assisted Transmission Power Control Solution for Energy-efficient Communications in WBAN," IEEE Journal on selected area in communications, pp. 3427-3437, Sep. 2016. Article (CrossRef Link)

[28] Y. Zhang and B. Zhang, "A relay-aided Transmission Power Control Method in Wireless Body Area Networks," IEEE Access, pp. 8408-8418, April 2017. Article (CrossRef Link)

[29] W. Zang and Y. Li, "Gait Cycle Driven Transmission Power Control Scheme for Wireless Body Area Network," IEEE Journal of biomedical and health informatics, pp. 1-10, March 2017. Article (CrossRef Link)

[30] Y. Li, L. Kong, F. Wu, Z. Zheng, and G. Chen, "MAPM: Movement-based Adaptive Prediction Mechanism for Energy Conservation in Body Sensor Networks," IEEE Globecom, pp. 1-6, Feb. 2017. Article (CrossRef Link)

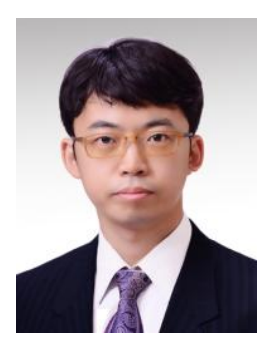

Woosik Lee received the B.S. degree in Computer Science from the Kyonggi University, Korea, in 2009, and the M.S. degree and the Ph.D. degree in the Computer Science from Kyonggi University in 2011 and 2016. In 2016, he was a research member of Korea Institute of Civil engineering and building Technology. In 2017, he was an assistant professor at the department of computer science, Kyonggi University, Korea. Since 2018, he has been a vice researcher at Social Security Information Service. His research interests include wireless systems, sensor networks, internet of things, and energy management protocols.

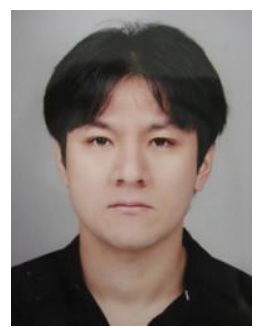

Heeyoul Kim received the B.E. degree in Computer Science from KAIST, Korea, in 2000, the M.S. degree in Computer Science from KAIST in 2002, and the Ph.D. degree in computer science from KAIST in 2007. From 2007 to 2008, he was a senior engineer of the Samsung Electronics. Since 2009 he has been a faculty member of Division of Computer Science at Kyonggi University. His main research interests include cryptography \& security such as secure group communication and clod computing security. 


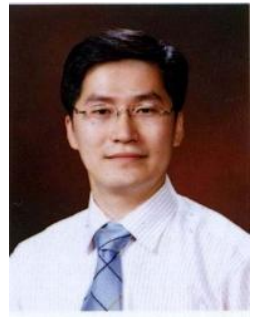

Min Hong received his B.S. from Soonchunhyang University, M.S. from University of Colorado at Boulder, and Ph.D. received from University of Colorado at Denver and Health Sciences Center in 1995, 2001, and 2005 respectively. Since 2014, he has been a faculty member of Computer Software Engineering in Soonchunhyag University. His research interests are in computer graphics, physically-based modeling and simulation, bioinformatics, and image processing related applications.

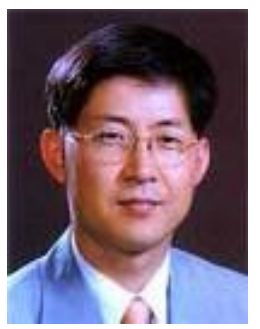

Min-Goo Kang is a professor in the dept. of IT contents at Hanshin University, Osan South Korea from 2000. He has received the B.S., M.S., and Ph.D. degrees from Yonsei University, Seoul, Korea all in Electronic Engineering in 1986, 1989 and 1994, respectively. He was a research engineer at Samsung Electronics from 1985 to 1997. His research interests include wireless communication algorithm, mobile devices, and Smart UX for Mobile TV \& DTV.

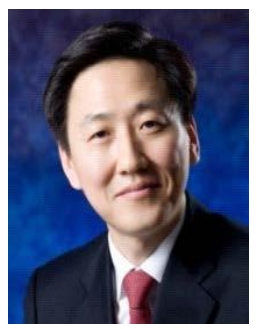

Seung Ryul Jeong is a Professor in the Graduate School of Business IT at Kookmin University, Korea. He holds a B.A. in Economics from Sogang University, Korea, an M.S. in MIS from University of Wisconsin, and a Ph.D. in MIS from the University of South Carolina, U.S.A. Professor Jeong has published extensively in the information systems field, with over 60 publications in refereed journals like Journal of MIS, Communications of the ACM, Information and Management, Journal of Systems and Software, among others.

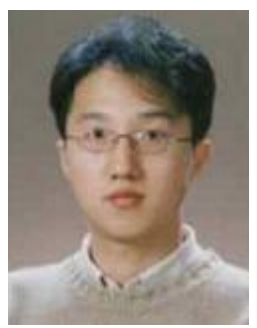

Namgi Kim received the B.S. degree in Computer Science from Sogang University, Korea, in 1997, and the M.S. degree and the Ph.D. degree in Computer Science from KAIST in 2000 and 2005, respectively. From 2005 to 2007, he was a research member of the Samsung Electronics. Since 2007, he has been a faculty of the Kyonggi University. His research interests include sensor system, wireless system, cloud computing, SDN, and mobile platform. 